\title{
Self and Interpersonal Communication
}

\author{
Prabhakararao Sampathirao ${ }^{1 *}$
}

\section{ABSTRACT}

One of the main things impacted by our self-concept and our self-esteem is our communication with other people. Self-concept, self image, self-esteem self efficacy are major factors in the way we communicate. Whether we are introverts or extroverts that can be seen in the way we communicate with others. Communication becomes smooth when we become part of it. People with high self esteem are confident, responsible, committed to goals, genuine and forgiving. An artificially inflated self-esteem is an effort to appear to have high self-esteem. However, such individuals don't typically show the characteristics of people with high self-esteem. Whereas low esteemed people insecure, unhappy and impatient. An artificially inflated self-esteem is an effort to appear to have high self-esteem. However, such individuals don't typically show the characteristics of people with high self-esteem. There are certain ways improve ones self-esteem. Development of a relationship is closely related to systematic self-disclosure. General personality traits such as quietness, shyness, and reticence frequently precipitate Communication Apprehension. Prevention and treatment methods of communication apprehension are now available.

Keywords: Self, Interpersonal Communication.

\section{Self-concept and communication}

The way we communicate is greatly influenced by our self-concept especially self-esteem. Only because of this influence some of us introverts, some others are extroverts and still some others are mixture of both. If somebody focuses on his or her sense of humor in his/her talks, he will be seen as a funny person by all around him whether or not he is aware of it. Our emotional needs and desires dictate our communication. If a person perceives himself as an introvert, but he or she doesn't like to be attributed so as it negatively affect his /her self-esteem and he may really want to be a funny person, he reassures himself again and again having a great sense of humor and he goes on to great lengths and breadths to tell jokes and try to amuse others.

Before going into the detailed discussion about our own communication forms, we need to understand certain basic terms and their association with development of our communication

\footnotetext{
${ }^{1}$ Health education Officer, Central health Education Bureau (CHEB) New delhi

*Responding Author

(C) 2016 I P Sampathirao; licensee IJIP. This is an Open Access Research distributed under the terms of the Creative Commons Attribution License (http://creativecommons.org/licenses/by/2.0), which permits unrestricted use, distribution, and reproduction in any Medium, provided the original work is properly cited.
} 


\section{Self and Interpersonal Communication}

habits. These are self-concept, self-image and self-esteem. Communication become smooth when we become part of it, being a part of it doesn't mean only our physical involvement, but attributes of our self-e.g., how we see ourselves, what type of feelings and thoughts we hold about our strengths and weaknesses. Relatively what kind of sustained perceptions we hold about ourselves, our emotional states, talents our likes, dislikes values and roles etc., how we want others to see us, how we think others should see us and how we want to see ourselves. All these attributes determine our communication habits. Most of our self-concept is a reflection of our relationships and the people around us. Our parents, near relatives, friends, heroes and bullies, all have influenced the way we perceive ourselves.

\section{How does self-concept affect our communication?}

Our self-concepts continuously develop and re-develops through constant reflection of our own images through others by variety of processes like looking glass self (1) reflected appraisal, ego boosters and busters; social comparisons (superior/inferior, same /different); cultural teachings and our own interpretations and experiences. Cultural influences on self-concept differ greatly from individualistic cultures to collectivistic cultures. In individualistic cultures just like in western cultures, self is separate, unique from other individuals and develops into independent, self-sufficient and without botheration about interdependence and place more emphasis on individual decisions and values, autonomy, youth, change, individual security and equality. These cultures reward and value individual achievements and blame individuals for failures. On the other hand, collectivistic cultures, like in oriental ones promote 'we' orientation and emphasize extended families, their care and in group living. Groups share credit as well as blame and reward contribution to group goals and group decisions, duty, order, tradition, age, group security, status and hierarchy. The five characteristics of self-concept are that it affects communication. It is subjective, resists change, multi-dimensional and flexible.

Our relationships with other people mainly affect two areas of our self-concept. i.e., our selfimage, or characteristics or traits we believe that we possess, and our self-esteem or our evaluation of what is worthwhile or valuable about ourselves. While our self-image is the descriptive side of our self-concept, the self-esteem is the evaluative side, which means the side that assigns value and worth to those traits and characteristics. The way in which I perceive myself is my self-concept within which my self-image perceives that I am below average handsome and a good writer. My self-esteem places more value on my writing skills than my physical personality as a positive aspect to which I belong. This entire process is a subconscious reflex that can cross into conscious awareness, but it's happening all the time throughout our lives. Self-concept, especially self-esteem, is a major factor in the way of our communication. Our communication may also directed by our self-esteem and self-concept, because it can often feel forced or unnatural, since it may not be that person's natural communication style. People can lose sight of their natural communication habits by trying to make up for what they see as a deficiency in their personality. 


\section{Self and Interpersonal Communication}

\section{Distortion of self-concept}

Self concept is derived from self esteem and self efficacy, but previous experiences may distort one's self concept as self assessment may not always accurate. If a person has low self esteem, the self-concept may be skewed in the negative direction. People with good self esteem and efficacy are often able to recognize their limitations without any judgment attached to it (2). The self-concept is subjective, which means it is subjected to obsolete, half-baked information and can be distorted after the subjective feedback, too much emphasis on perfectionism. Social expectations like modesty or egotism also affects self-concept. Self-concept is conservative and resists change and tends to cling to present even when evidence shows that it is outdated, cling to negative outmoded self-perception whether it is positive or negative and therefore, result in selfdelusion and lack of growth. A healthy self-concept is flexible and subject to change like any other phenomena, therefore, we need to constantly adjust our self-concept according to the changes that are taking place in our surroundings. In the course of changing our self-concept, we should have will to change and develop necessary skills to change and cultivate realistic expectations and always strive for such realistic perception (4).

Self-concept operates at multi-dimensional levels. If there is a mismatch between how you see yourself (e.g. yourself image) and what you'd like to be (e.g. your ideal self) then this is likely to affect how much you value yourself. Therefore, there is an intimate relationship between selfimage, ego-ideal and self-esteem. A person's ideal self may not be consistent with what actually happens in life and experiences of the person. Hence, a difference may exist between a person's ideal self and actual experience. This is called incongruence. Where a person's ideal self and actual experience are consistent or very similar, a state of congruence exists. All persons experience a certain amount of incongruence as in reality rarely does a total state of congruence that ever exists. The development of congruence is dependent on unconditional positive regard. For a person to achieve self-actualization they must be in a state of congruence (3). There are four major factors which influence its development firstly, the ways in which others (particularly significant others) react to us. Secondly, how we think we compare to others, thirdly, our social roles, finally, the extent to which we identify with other people (5)

Self concept has three components i.e., the view that person has for himself i.e., self- image; the value a person place upon himself is self- esteem and it always involves a degree of evaluation and we may have either a positive or a negative view of ourselves (3). A person's self image does not necessarily have to reflect reality and it is affected by many factors, such as parental influences, friends, the media etc. Self-concept is also derived from self-esteem and self efficacy. Self -esteem is the regard or respect a person has for himself and a person with positive feelings is said to have high self- esteem. However, self- esteem can refer to very specific areas as well as general feelings about self. For example, a person may have low self esteem about his physical attractiveness but high self esteem of doing a job well. In uncertain or anxiety arousing situations our self-esteem may change rapidly (6) 


\section{Self and Interpersonal Communication}

There are four major factors that influence self esteem and they are the ways in which others (particularly significant others) react to us, while affirmative reactions develop a positive selfimage and reactions of avoidance develop into negative self- image. How we think we compare to other, if the people we compare with ourselves (our reference group) appear to be more successful, happier, richer, better looking than ourselves we tend to develop a negative self image BUT if they are less successful than us our image will be positive. People in Some social roles carry prestige e.g. doctor, airline pilot, TV presenter, premiership footballer and this promotes self-esteem. Other roles carry stigma. E.g., prisoner, mental hospital patient, refuse collector or unemployed person. The extent to which we identify with other people also become part of our personality i.e. we identity with the positions we occupy, the roles we play and the groups we belong to (7). But just like all these other factors, the influence of our parents is equally important (8).

The ideal- self is what the person cherish to be like .If there is a mismatch between how a person see himself (e.g. self image) and what he'd like to be (e.g. ideal self ) then this is likely to affect how much he value himself (self-esteem). Therefore, there is an intimate relationship between self-image, ego-ideal and self-esteem. A person's ideal self may not be consistent with what actually happens in his life and his own experiences. Hence, a difference may exist between a person's ideal self and actual experience. This is called incongruence; rarely does a total state of congruence exists as every person experience certain amount of incongruence. Where a person's ideal self and actual experience are consistent or very similar, a state of congruence exists. The development of congruence is dependent on unconditional positive regard. For a person to achieve self-actualization they must be in a state of congruence (3). One needs high self-esteem in order to self-actualise and realise one's full potential.

\section{Ego and its affect on communication:}

The ego is the opposite of self-esteem. The problem with the ego is it can 'disguise' itself as your self-esteem and it is important to become aware of this behaviour when it arises. The most commonly agreed up on definition of ego is it is your self-defense mechanism and more importantly, your false concept. All the ego is concerned with indulging in self-destructive behaviours and differentiating itself from others, whether it is talking about other people behind their back (out of insecurity) or self-appraisal (beating ones own drum). The ego needs to be validated at all costs in order to 'survive' if it is not weaken.

The ego is like an inner child, constantly need attention and if it does not receive it, lashes out. Egocentricity is very common in Pick Up, most men are very egocentric when it comes to their dating lives, This kind of behaviour can also be seen with materialistic people; buying more and more possessions to fuel their false self-concept. Face book has become a haven for the ego driven; statuses are often nothing but false self-esteem increasers with each person racking up 'likes' to differentiate themselves from others. The ego loves to feel what is called 'otherness' from others. However, the ego can also be self-destructive; it can make you feel worthless, 


\section{Self and Interpersonal Communication}

lonely, depressed, and insignificant and all those other negative emotions. When you catch yourself having egocentric thoughts, become present; do not judge or condemn the thought (if you do this it will argue back) and just become aware of it, it will soon dissipate. To paraphrase Tolle, "once you bring it into the light of consciousness, the ego is unable to 'survive' (21)."

\section{Artificial inflated self esteem and true self esteem:}

In order to define the characteristics of high self-esteem, there is need to make a distinction between an artificially inflated self-esteem and true self-esteem. An artificially inflated selfesteem is an effort to appear to have high self-esteem. However, such individuals don't typically show the characteristics of people with high self-esteem. Individuals with high self-esteem have the following characteristics in abundance with consistency. 1) Responsibility: Since individuals with high self-esteem can accept themselves completely they are able to take responsibility for themselves and the consequences of their actions without being excessively critical of themselves. Therefore, they are readily able to acknowledge mistakes and accept limitations. 2) Goal commitment: Those with high self-esteem tend to have a strong sense of purpose and are committed to goals in life. In addition, they tend to be persistent in achieving these goals as their commitment does not fluctuate based on success or failure. As active participants in life they tend to strive for excellence not for perfection.3) Genuineness.: People with high self-esteem can be honest with themselves and others both emotionally and intellectually. As they aren't fearful of others truly knowing them, they tend to be genuine in their interactions with others. 4) Forgiving: High self-esteem corresponds with high degree of tolerance and acceptance of limitations and they easily forgive themselves and others 5) Internal values: Individuals with high self-esteem tend to have internally-based values rather than externally-based. In other words, they have a strong identity based on chosen values rather than values they believe due to the demands or expectations of others. This type of identity is usually considered an "achieved identity" in which a person has analyzed their beliefs and values to decide the set of internal principles or values that they will adhere to. 6) Positivity: People with high self-esteem are positive with an appreciative and grateful attitude towards life. They can freely praise themselves and others and tend to look for the positive aspects of life and not dwell on the negative.7) Self-improvement: Generally, there is a strong tendency to strive towards selfimprovement among those with high self-esteem. As they don't view the need for selfimprovement as a negative quality they are able to examine themselves uncritically. In addition, they can ask for help as needed because they don't view the need for help as shameful or negative.

The characteristics of low self-esteem are feelings of unhappiness and sometimes it may lead to depression, feelings of anxiety especially social anxiety which is a consequence of social evaluative aspect of self esteem based on comparisons with other people. Feelings of inferiority or superiority: most people who have low self- esteem feel inferior to others and they consistently believe that there is some flaw within them because of which they are unable to meet certain standards which others have met. Sometimes this perceived flaw is more magnified 


\section{Self and Interpersonal Communication}

by the person himself than visible to others because of his past experiences. Some people with low self esteem with or without perfectionist tendency due to concern about what others may think of them present an air of superiority in a bid to cover how they truly feel about themselves.

Impatience or irritation with self or others: most frequently the persons with low self esteem get impatient or irritated with flaws, mistakes or inadequacies mostly directed towards themselves but rarely towards others. Externally oriented goals: individuals with low self esteem often determine goals and direction in life based upon what others might want or need, such an orientation may result in resentment due to taking care of only others needs but not their own. Negativity: low self esteem tends to leads to negativity which may not always external but also internal as well. External manifestations like criticizing oneself to others or excessively apologizing or commenting upon negative observations can be noticed by others. Unfortunately, people tend to avoid individuals who are excessively negative.

Self -efficacy is a person's belief in his ability to accomplish a specific goal or task and it is corresponds to an individual's level of competence which varies from situation to situation and as a result his assessment of overall self-efficacy may not be complete and accurate as it is an assessment of an individual's general feeling of competence across a variety of situations or tasks.

\section{Characteristics of low self-efficacy:}

1) Fear of risks: Individuals with low self-efficacy always see themselves as unable to be successful and they are often unwilling to take risks or try new things because they are convinced that the result will be a definite failure. This is particularly unfortunate because the main way to increase self-efficacy is through practice and experience. 2) Fear of uncertainty: Low selfefficacy often is related to self-doubt and uncertainty. The individual doesn't want to try without a guarantee of success and therefore, they may never discover things at which they could be successful. 3) Feelings of failure: Those with low self-efficacy frequently have feelings of failure and hence avoid or remain untried new things in their life due to the risks involved. Or, they might only try something half-heartedly and therefore, they are less likely to experience success and more likely to see a failure. 4) Impression management: Impression management is the attempt to control how others might perceive you in order to be seen more positively. People with low self-efficacy feel they are not capable but may try to present a successful and competent image to others. They may put a great deal of energy into behaving in a way to obtain approval from others and experience a great deal of worry about being found out to be a fraud. For instance, they may try to hide mistakes from others rather than learn from them which prevents them from increasing their sense of self-efficacy.

\section{Characteristics of high self efficacy:}

1) Self-confidence: One of the most obvious characteristics of high self-efficacy is selfconfidence. They approach tasks or situations with a sense of their ability to be successful. This 


\section{Self and Interpersonal Communication}

self-confidence tends to lead to more experience which increases their ability which leads to greater self-confidence. This positive cycle lends itself to increasing self-efficacy even further. 2) Accurate self-evaluation: Individuals with high self-efficacy tend to be able to accurately evaluate their performance. They are neither overly-critical nor overly positive but are able to examine themselves realistically in order to pursue self-improvement. 3) Willingness to take risks: Those with high self-efficacy are willing to take risks because they understand that taking calculated risks increases the chances of success. As they are not fearful of failure or mistakes, reasonable risks can only increase self-efficacy. 4) Sense of accomplishment: Generally those with high self-efficacy feel a sense of accomplishment because they are often more successful due to the willingness to take risk and to pursue interests. Even if they fail or make mistakes they feel a sense of accomplishment because they view mistakes as opportunities to improve themselves.

\section{Good sense of self-efficacy but low self-esteem?}

Self-efficacy and self-esteem are similar concepts but they are not the same thing. They do tend to correspond so that a person who is low in one is more likely to be low in the other. But it is also possible to have low self-esteem and yet have high self-efficacy. In fact, a lot of perfectionists show this combination frequently. Therefore, someone may tend to be overlycritical and negative about him and yet see himself as quite capable in certain areas. For instance, he might see himself as uninteresting and unlikeable but see himself as a competent architect. This occurs frequently with perfectionists because they are often competent at tasks with clear guidelines but feel uncertain in situations without clear "rules" such as relationships.

\section{How can self-esteem be improved?}

1) Eliminate negative self-talk: First and foremost, people with low self-esteem need to eliminate harmful self-talk. The negative labels and frequent self-criticism can only cause further damage. Eliminating negative self-talk doesn't mean you can't recognize and address problems, but it means to be careful about how you talk to yourself and to not be self-destructive. 2) Recognize strengths: Those with low self-esteem tend to focus on their weaknesses rather than focusing on their strengths sometimes claiming that there isn't anything positive they can say about themselves which is totally untrue. It is important to pay attention to strengths and to appreciate the strengths no matter how small they may seem. Once you recognize the strengths you need to reinforce the strengths through frequent focus on them. 3) Recognize self-worth: It is important to recognize that every person is a unique human being and have worth. Recognizing that one deserves to take care of himself and set limits and to reinforce this idea by continuing to focus on self-worth. 4) Accept mistakes: Recognize that mistakes and flaws are part of the human condition and don't make the committed person less than others. Instead, make equal with others. One may have flaws and make mistakes. The more actively we are involved in life, the more mistakes we will make. But being actively involved allows us more opportunity for success as well. Accept self-flaws and all. 5) Accept rejection: The more we believe that everyone doesn't have to like us, the less we need to feel bad or be ashamed of our imperfections. No one 


\section{Self and Interpersonal Communication}

can be liked by everyone! It is an impossible task. However, the person with low self-esteem often feels a failure if someone is disapproving or rejecting. Instead, he should congratulate himself if someone doesn't like him because he is a genuine person.

\section{How to improve self -efficacy}

1) Develop skill set: The most important way to improve self-efficacy is to develop the skill set that needed to be effective. If problem is the success at work, identify the areas of deficit and determine what is needed improve. Ask others to honestly evaluate skills and to give specific advice regarding improvement. Once it is known what is needed, do it again and again until you feel competent. That's how competence develops. People aren't born with competence; they have to learn and practice in order to become competent. 2) Modelling: One way to learn the necessary skills is to observe others. You can observe successful completion of tasks to learn how to achieve success. When you observe others being rewarded for their performance or successful completion of a task, you are more likely to be able to model yourself after their behavior. 3) Focus on specifics: To improve self-efficacy, it is best to focus on specifics. If someone gives you general feedback especially if it is negative you are less able to make changes than if someone can provide specific feedback. For instance, if you want a child to learn how to do dishes you don't say "These dishes aren't clean," instead you say "Let me show you how to load the dishwasher to get the best results.” 4) Reinforcement: The more behavior is reinforced, the more likely it will continue. If you want to improve your self-efficacy focus on what you do well and reinforce it by giving yourself specific praise.

\section{Positive thinking is no change in thinking.}

The problem with artificial positive thinking is that it is not believable; therefore it cannot change the self-esteem. The main challenge of working with people with low self-esteem is to develop believable statements. So the challenge of improving self-esteem is to develop believable statements. Like "I'm a person who is willing to learn about myself and make improvements" or "I have courage because I am facing something that is very difficult for me" or "I am persistent. Even though happiness has eluded me I keep trying." Notice with these statements there are specifics attached to them. They are not general and overly positive. Instead, the statements are realistic with specific reasons why they are true. To change self-esteem, it is necessary to create these types of statements.

\section{Self-awareness:}

Johari window (9) is a simple and useful tool for understanding self-awareness with reference to self and others. Here self means the a person in group or team and others means other persons in the team or group. The four Johari window perspectives are called regions, areas or quadrants. Each represents information, the first quadrant represents open self that is known by the person about himself/herself and is also known by the others. The second quadrant represents blind self which is unknown by the person himself/herself but other people knew. It also third quarter represents hidden self what is known by the person himself/ herself but other people do not 


\section{Self and Interpersonal Communication}

know. The fourth quarter represents unknown self that is unknown by the person about himself/herself and also by the others. The aim is to increase the open self (area) by reducing blind area through feedback, by reducing hidden area by self-disclosure.

\section{Self-disclosure}

Development of a relationship is closely related to systematic exchanges in communication. Relationships generally begin with the exchange of superficial information and gradually move on to more meaningful conversations. In order to develop a more intimate relationship, partners must increase the breadth and depth of their conversations. Breadth includes the variety of topics two people discuss and depth is the personal significance of these topics (social penetration theory).this can be explained with an example of a wedge. The beginning of a relationship is represented by a narrow and shallow wedge because only a few topics are discussed. However, as the relationship goes on, the wedge should become broader and deeper, including more topics of personal significance. The wedge must drive through three "layers" in order for intimacy to develop. The first is superficial "small talk" with little personal information about the speakers. The next layer is intimate, with increasing breadth and depth and more personal details. The third is the very intimate level, where extremely private information is shared (10). Intimacy in these relationships can develop only if the persons involved reciprocate disclosures. Reciprocity must be gradual and match the intimacy of the other's disclosures.

\section{Factors influencing self-disclosure:}

The first is intra-individual factors that are on the child's mind and cause him or her to need social input. Biological development, cultural and social pressures, and individual maturity determine these issues, and, thus, a child's age, personality, and background also contribute to his or her level and need of self-disclose in a relationship with a parent. Second set of factors is called contextual factors, which include the opportunities and situations that the individual has to disclose as created by the socio-cultural environment. Girls are noted for usually disclosing their problems, mostly to their mothers, while boys reveal more about bad grades, behavioral conflicts, and other issues to both parents. Certain people called high openers more likely to get others to disclose. Even people known to disclose very little are likely to disclose more to high openers. Thus, if parents are characterized as good listeners, trustworthy, accepting, relaxed, and sympathetic, as are high openers, then they will likely elicit more disclosure from their children. Feelings, positive or negative about the parent-child relationship during one's upbringing have also been found to correlate with the child's disclosures to the parents. Sometimes children qualify their disclosures by merely stating that they only disclose what they feel they want to their parents. Thus, some information is kept secret. This is dubbed selective self-disclosure.

\section{Risks and rewards of self- disclosure:}

Rewards: self-disclosure enable to know the self-better, improve physiological health and increase communication better. The self-disclosure of children to their parents is the main source of information to the parents to gain knowledge about their children and their daily lives. 


\section{Self and Interpersonal Communication}

The more parents know about their kids, the lower the rate of behavior problems among children, and the higher the children's well-being. Adolescents who disclose have been found to have lower rates of substance abuse, lower rates of risky sexual behaviors, lower anxiety levels, and lower rates of depression (11). The risks of self-disclosure include personal risks, relational risks and professional risks (12).

\section{Self-disclosure on the Internet}

There are four major differences between online communication and face to face communication. The first is that Internet users can remain anonymous. The user can choose what personal information (if any) they share with other users. Even if the user decides to use their own name, if communicating with people in others cities or countries they are still relatively anonymous. The second is that physical distance does not limit interaction on the Internet the way it does in real life. The Internet gives the ability to interact with people all over the world and the chance to meet people who have similar interests that one may not have met in their offline life. Visual cues, including those pertaining to physical attractiveness, are also not always present on the Internet. These factors have been shown to influence initial attraction and relationship formation. Finally, Internet users have time to formulate conversations which is not allotted in face to face conversation. This gives a user more control in the conversation because they do not have to give an immediate response (13)

\section{Communication apprehension:}

Communication apprehension is defined as an individual level of fear or anxiety associated with either real or anticipated communication with another person or persons. General personality traits such as quietness, shyness, and reticence frequently precipitate Communication Apprehension. When the ability and desire to participate in discussion are present, but the process of verbalizing is inhibited, shyness or reticence is occurring (14). The degree of shyness, or range of situations that it affects, varies greatly from individual to individual. Seven factors have been identified as causing communication apprehension. These are low intellectual skills, speech skill deficiencies, voluntary social introversion, social alienation, communication anxiety, low social self-esteem and ethnic/ cultural divergence in communication norms. According to Negative Cognitive Appraisal Model (15). Where a quiet child on being criticized for his or her early language performance, learned to expect negative reactions and subsequently learned to avoid them by keeping quiet. If the teachers, parents, or other children always reacting negatively towards a child's talk, the child will perform poorly and avoid oral communication situations (16). In adults it affect their degree of evaluation, subordinate status, degree of conspicuousness, degree of unpredictability, degree of dissimilarity, prior success and failure and lack of communication skills and experience.

\section{Consequences of communication apprehension:}

The consequences of Communication Apprehension are emotional, educational, and social. Shyness and reticence affect the social skills necessary for children to make friends. Shy students 


\section{Self and Interpersonal Communication}

tend to confine their career aspirations to vocations that require little oral communication. They seem to have a higher need to avoid failure, and they have less achievement or success motivation than other students. The teacher may regard quiet students as "perfect" in the classroom as they are not discipline problems. But often the CA students' lack of response or participation has a negative, spiralling affect--they are perceived as less capable, and are thus called on less frequently in class discussion. Their lack of enthusiasm tends to limit teachers' attention to them, which further reinforces their own self-evaluation (17). In the adults Communication Apprehension results in decrease in frequency, strength and likelihood of engaging in communication, least likely to volunteer, avoid leadership due to talking/disclosing less, avoid jobs requiring communication, less likely to get job interviews and lack of satisfaction in dating.

Much of the communibiological research done on communication apprehension has found that the causes of the affliction are inherited..Primary components of communication apprehension to be introversion and neuroticism and activation of the behavioral inhibition system is related to anxiety (18). People with low communication apprehension communicate for pleasure, affection, control, and relaxation while people with high communication apprehension communicate for inclusion and escape. Communication apprehension in the first language predicted communication apprehension in the second language. Although, the basis of a person's communication behavior is based on genetics, a good portion of their behavior is also affected by the culture they are raised in. In fact, the communication behaviors ethnocentrism and homophobia were found to have no relationship with a person's genetic make-up or temperament.[11] These traits are instead developed through culture. Apprehension increases with unfamiliarity, more and more stereotypes and prejudices in the culture. Situational influences includes ambiguity, status and perception of evaluation.

Prevention and treatment of communication apprehension:

To address the problem of CA on a somewhat broader level, (16) proposes a four-phase strategy where teachers in training should take more than an introductory course in oral communication (e.g., a course aimed at understanding the communication behaviors of students, create basic communication courses in the earlier elementary grades, to provide specialized treatment for quiet/shy students on a voluntary basis and develop classroom activities that encourage oral communication

Treatments include cognitive restructuring which involve changing unrealistic beliefs which cause fear of failure. The most commonly used treatment for CA in adults has been "systematic desensitization to unlearn apprehension creating hierarchy behaviours leading up to desired behaviours" This includes training in deep muscle relaxation, construction of anxiety-creating stimuli, and the graduated pairing, through imagery, of these anxiety stimuli with the relaxed state (19). Variations of these methods may also be effective for younger students (20). 
Acquiring skills like preparing and practicing, focussing on success, familiarizing with situation and relaxing. Preventing or alleviating communication apprehension for every student is a monumental if not impossible task. But simply recognizing that CA is a frequent phenomenon that often occurs early in students' lives can be a spur toward eliminating many factors that contribute to the quiet child's withdrawal from communication.

\section{CONCLUSION:}

Our communication style and inner self are mutually dependent and affect each other. Since our self evolve in different phases in our life it needs to be nurtured through the healthy socialisation process to achieve all round development.

\section{REFERENCES:}

1. Theory of looking glass self: Cooley.

2. The pillars of the self -concept : self esteem and self efficacy by Monic A. Frank, Ph.D.

3. Rogers, C. (1959). A theory of therapy, personality and interpersonal relationships as developed in the

client-centered framework. In (ed.) S.

4. Self in Interpersonal Communication Chapter 3, Part 1 Devito 10 th ed .

5. Argyle, M. (2008). Social encounters: Contributions to social interaction. Aldine Transaction.

6. Morse, S. J. \& Gergen, K. J. (1970). Social comparison, self-consistency and the concept of self. Journal of Personality and Social Psychology, 16, 148-156.

7. Argyle, M. (2008). Social encounters: Contributions to social interaction. Aldine Transaction

Coopersmith, S. (1967). The antecedents of self-esteem. San Francisco: Freeman.

8. Johari window : developed in 1955 by Joseph Luft and Harry Ingham calling it Johari after combining their first namesJoe and Harry.

9. Altman, I., \& Taylor, D. A. (1973). Social penetration: The development of interpersonal relationships. New York: Holt, Rinehart \& Winston.

10. Urry, S. A., Nelson, L. J., \& Padilla-Walker, L. M. (2011). Mother knows best: Psychological control, child disclosure, and maternal knowledge in emerging adulthood. Journal of Family Studies, 17(2), 157-173. doi:10.5172/jfs.2011.17.2.157.

11. Sturges, J. W. (2012). Use of therapist self-disclosure and self-involving statements. The Behavior Therapist, 35(5), 90-93.

12. McKenna, Katelyn Y. A.; Bargh, John A. (2000). "Plan 9 From Cyberspace: The Implications of the Internet for Personality and Social Psychology". Personality and Social Psychology Review 4 (1): 57-75.doi:10.1207/s15327957pspr0401_6.

13. Friedman, P. G. "Shyness and Reticence in Students." Washington, D.C.: National Education Association, 1980. Stock No. 1675-0-00. ED 181520 


\section{Self and Interpersonal Communication}

14. Glaser, S. R. "Oral Communication Apprehension and Avoidance: The Current Status of Treatment Research." COMMUNICATION EDUCATION 30 (1981): 321-41).

15. Glaser, S. R. "Oral Communication Apprehension and Avoidance: The Current Status of Treatment Research." COMMUNICATION EDUCATION 30 (1981): 321-41).

16. Richmond, V. "Implications of Quietness: Some Facts and Speculations." In AVOIDING COMMUNICATION: SHYNESS, RETICENCE, AND COMMUNICATION APPREHENSION. J. A. Daly and J. C. McCroskey, eds. Beverly Hills: Sage Publications, 1984).

17. McCroskey, J. C. "Quiet Children in the Classroom: On Helping Not Hurting." COMMUNICATION EDUCATION 29 (1980: 239-44.

18. Friedrich, G., and B. Goss. "Systematic Desensitization." In AVOIDING COMMUNICATION: SHYNESS, RETICENCE, AND COMMUNICATION APPREHENSION. J. A. Daly and J. C. McCroskey, eds. Beverly Hills,Calif.: Sage Publications, 1984.

19. Communication Apprehension: The Quiet Student in Your Classroom. ERIC Digest.

20. Eckhart Tolle's 'The Power of Now'

How to cite this article: P Sampathirao (2016) Self and Interpersonal Communication, International Journal of Indian Psychology, Volume 3, Issue 3, No. 5, DIP: 18.01.086/20160303 